\title{
Rosa Anna Greco, Blancandin et l'Orgueilleuse d'amours. Versioni in prosa del XV secolo
}

\section{Maria Colombo Timelli}

\section{(2) OpenEdition}

1 Journals

\section{Édition électronique}

URL : http://journals.openedition.org/studifrancesi/35787

DOI : 10.4000/studifrancesi.35787

ISSN : 2421-5856

Éditeur

Rosenberg \& Sellier

\section{Édition imprimée}

Date de publication : 1 juillet 2005

Pagination : 138-139

ISSN : 0039-2944

\section{Référence électronique}

Maria Colombo Timelli, « Rosa Anna Greco, Blancandin et l'Orgueilleuse d'amours. Versioni in prosa del XV secolo ", Studi Francesi [En ligne], 145 (XLIX | I) | 2005, mis en ligne le 30 novembre 2015, consulté le 20 avril 2021. URL : http://journals.openedition.org/studifrancesi/35787 ; DOI : https://doi.org/ 10.4000/studifrancesi.35787

Ce document a été généré automatiquement le 20 avril 2021.

\section{(c) $(1) \ominus$}

Studi Francesi è distribuita con Licenza Creative Commons Attribuzione - Non commerciale - Non opere derivate 4.0 Internazionale. 


\title{
Rosa Anna Greco, Blancandin et l'Orgueilleuse d'amours. Versioni in prosa del XV secolo
}

\author{
Maria Colombo Timelli
}

\section{RÉFÉRENCE}

ROSA ANNA GRECO, Blancandin et l'Orgueilleuse d'amours. Versioni in prosa del XV secolo, Alessandria, Edizioni dell'Orso, 2002 («Bibliotheca Romanica. Studi e Testi», 3), pp. 297.

L'histoire de Blancandin et de l'Orgueilleuse d'amours est conservée d'abord sous la forme d'un roman en vers (un peu plus de 6000 octosyllabes à rimes plates), composé par un auteur anonyme vers 1200-1230. Ce roman d'aventures, transmis par cinq manuscrits complets et par deux fragments d'un sixième, réunit des thèmes purement romanesques (la source principale se reconnaît facilement dans Cligés et dans Perceval de Chrétien de Troyes), des motifs épiques, et des thèmes hagiographiques. R. A. Greco consacre la première partie de son Introduction à cette source, en reprenant les résultats des études précédentes, et en corrigeant en partie le stemma codicum de F. P. Sweetser, éditeur du texte en vers. Elle passe ensuite à la présentation de la version en prose, exécutée en milieu bourguignon sans doute vers 1450. Cette adaptation est conservée par trois manuscrits contemporains, appartenant à deux familles: le manuscrit $\mathrm{Br}$ (Bruxelles, B.R. 3576-3577), qui contient une version plus simple et résultant souvent d'une opération de «dérimage», et les deux autres ( $V$ : Vienne, Osterreichische Nationalbibliothek, 3438; et Pa: Paris, BnF, fr. 24371), qui offrent une rédaction plus ample bien que dépendant du même archétype que $\mathrm{Br}$. Comme cela est le cas pour d'autres proses bourguignonnes de l'époque, l'amplification s'effectue essentiellement par une forme plus diffuse, grâce à des procédés stylistiques bien connus (redoublements synonymiques, imbrication syntaxique etc.); ce n'est que dans la 
dernière partie du roman (après le ch. 24) que la version de $V$ et de $P a$ s'enrichit de nombreux épisodes de guerre et s'éloigne donc partiellement de $B r$.

Une collation détaillée montre clairement les différences entre les deux familles, alors que la dérivation $V>P a$, donnée pour acquise dans le stemma (p. 40), n'est pas traitée explicitement. La discussion des rapports entre les trois manuscrits s'arrête là, sans que le choix éventuel d'un manuscrit de base (soit $\mathrm{Br}$ ou $\mathrm{V}$ ) soit pris en compte. Une troisième partie de l'Introduction étudie le travail du remanieur, travail dépourvu d'originalité réelle, et limité au fond aux procédés habituels, répondant aux goûts littéraires et esthétiques des destinataires: création d'un prologue, organisation en chapitres, procédés d'actualisation culturelle, amplification et/ou suppression d'épisodes et de personnages secondaires. Quelques aspects du remaniement auraient peut-être mérité un peu plus d'attention, entre autres la facture du prologue: celui de $V$ (et de $\mathrm{Pa}$ ) indique explicitement le commanditaire de l'œuvre: «a la requeste et priere de mon treshonnouré seigneur Jehan seigneur de Crequy et de Canaples, auquel vouldroie complaire» (édition, p. 150); celui de $B r$, manuscrit qui appartenait cependant à la librairie de Philippe le Bon (Barrois, n. 1302), se lit ainsi: "par commandement auquel n'oseroie ne vouldroie desobeir». Or, R. A. Greco déclare que ce prologue semblerait suggérer Philippe le Bon lui-même (p. 45), alors qu'il me semble beaucoup plus probable, quel que fût le destinataire indiqué dans l'archétype, qu'une lacune se soit produite entre «commandement» et «auquel». D'autre part, l'idée que cette prose de Bruxelles, qui consacre une place réduite aux scènes de bataille et supprime quelques personnages, soit destinée «a una fanciulla di rango elevato» (p. 54) me paraît tout au moins faible.

3 L'étude linguistique (pp. 55-63) relève les phénomènes caractéristiques de la scripta du moyen français du $\mathrm{XV}^{\mathrm{e}}$ siècle, avec quelques traits picardisants; menée sur les deux manuscrits $\mathrm{Br}$ et $V$, elle ne permet pas vraiment de reconnaître les péculiarités éventuelles de chaque copiste. La description des manuscrits occupe les pp. 63-69.

Suit l'édition des deux versions.

Quelques remarques d'abord sur le texte de $\mathrm{Br}$ (pp. 81-144). L'«auteur» organise le roman en trois parties, qu'il appelle «traittiés» (aussi bien dans la table que dans le texte): loin de désigner des «trattati» (cf. l'introduction, p. 41 et p. 63), ce terme, employé aussi dans le Cligés en prose avec la même acception, indique simplement les trois «parties» de l'œuvre; il aurait mérité soit un petit commentaire, soit d'être introduit dans le Glossaire. Quant au traitement du texte, la ponctuation est très sobre, parfois incorrecte (un seul exemple: 38,8 , où la valeur de «qui» = «si l'on» n'a pas été exactement interprétée, et où le point d'interrogation doit par conséquent être remplacé par une virgule); on remarque aussi un emploi irrégulier de ç (chevauca 12,8; couca 26,18; pença 30,8; presca 32,54; trenca 34,14 et 40,48). Le texte n'a pas toujours été compris; des corrections s'imposent, à mon avis, aux endroits suivants: 4,11 (garder que), 4,13 (couvert à remplacer par un passé simple, ainsi que dans $V$ 4,21), 4,23 (adoube, à lire adoubé), 5,37 (supprimer qui), 18,22 (signaler la lacune [...]), 18,45 (sy, à lire s'y), 19,10 (supprimer et), 32,43-45 (syntaxe à discuter), 40,36 (doleans, à lire de leans), 44,104 (dyplographie de aller). Au ch. 32, après la ligne 35, la numérotation des lignes est à revoir.

$6 \quad$ Le texte de $V$ se lit aux pp. 145-275. Sans entrer dans les détails (mêmes problèmes concernant la ponctuation de l'édition et certaines lectures à revoir), remarquons que le choix de publier les deux textes l'un à la suite de l'autre rend difficile - à certains 
endroits franchement impossible - la comparaison des deux versions: une simple table synoptique basée sur la division et sur le contenu des chapitres, sinon une édition en regard des deux versions, aurait permis de mieux rendre compte des modifications d'une rédaction à l'autre.

7 L'édition comprend les compléments habituels: des notes au texte, très sobres (Br: pp. 143-144; V: pp. 273-275), une liste des proverbes (pp. 277-278), à laquelle il faudrait ajouter $\mathrm{Br}$ 19,31: «il fault souffrir pour avoir mieulx»; la table des noms propres (pp. 279-281); un glossaire sélectif signalant les occurrences (pp. 283-295): on y relève souvent des définitions encyclopédiques plutôt que de véritables traductions (busines: 'strumenti musicali a fiato, piccole trombe'; coiffe: 'calotta di ferro sotto l'elmo'), ainsi que quelques traductions incongrues (habillemens: 'congegni bellici'; ravellee: 'rivelata'). 